University of Wollongong

Research Online

Faculty of Engineering - Papers (Archive)

Faculty of Engineering and Information

Sciences

2007

\title{
Solid State Microdosimetry With Heavy lons for Space Applications
}

\author{
A. Wroe \\ University of Wollongong, awroe@uow.edu.au \\ Anatoly B. Rosenfeld \\ University of Wollongong, anatoly@uow.edu.au \\ M. Reinhard \\ Australian Nuclear Science and Technology Organisation, Sydney \\ V. Pisacane \\ United States Naval Academy, USA \\ J. Zeigler \\ United States Naval Academy, USA
}

See next page for additional authors

Follow this and additional works at: https://ro.uow.edu.au/engpapers

Part of the Engineering Commons

https://ro.uow.edu.au/engpapers/428

\section{Recommended Citation}

Wroe, A.; Rosenfeld, Anatoly B.; Reinhard, M.; Pisacane, V.; Zeigler, J.; Nelson, M.; Cucinotta, F.; Zaider, M.; and Dicello, J.: Solid State Microdosimetry With Heavy Ions for Space Applications 2007.

https://ro.uow.edu.au/engpapers/428

Research Online is the open access institutional repository for the University of Wollongong. For further information contact the UOW Library: research-pubs@uow.edu.au 
Authors

A. Wroe, Anatoly B. Rosenfeld, M. Reinhard, V. Pisacane, J. Zeigler, M. Nelson, F. Cucinotta, M. Zaider, and J. Dicello 


\title{
Solid State Microdosimetry With Heavy Ions for Space Applications
}

\author{
A. Wroe, Student Member, IEEE, A. Rosenfeld, Senior Member, IEEE, M. Reinhard, Member, IEEE, V. Pisacane,
}

J. Ziegler, M. Nelson, F. Cucinotta, M. Zaider, and J. Dicello

\begin{abstract}
This work provides information pertaining to the performance of Silicon-On-Insulator (SOI) microdosimeters in heavy ion radiation fields. SOI microdosimeters have been previously tested in light ion radiation fields for both space and therapeutic applications, however their response has not been established in high energy, heavy ion radiation fields which are experienced in space. Irradiations were completed at the NASA Space Radiation Laboratory at BNL using $0.6 \mathrm{GeV} / \mathrm{u}$ Fe and 1.0 GeV/u Ti ions. Energy deposition and lineal energy spectra were obtained with this device at various depths within a Lucite phantom along the central axis of the beam. The response of which was compared with existing proportional counter data to assess the applicability of SOI microdosimeters to future deployments in space missions.
\end{abstract}

Index Terms-Heavy ions, microdosimetry, space.

\section{INTRODUCTION}

$\mathbf{T}$ ODAY the further exploration of space is a priority for world space organizations. Such exploration will lead to the exposure of both man and machine to different spectra of radiation than that experienced in near Earth environments and has led to various research both in physics and biology [1]-[5]. In deep space the radiation environment consists mainly of galactic cosmic radiation (GCR). With an energy range from $100 \mathrm{MeV}$ per nucleon to $10 \mathrm{GeV}$ per nucleon, the GCR consists of 87 percent protons, 12 percent helium ions, and 1 percent heavier ions [6]. Heavier particles are also present in near Earth environments the abundance of which is reflected in Fig. 1.

Heavy ions are an important factor for the consideration of biological effects on humans and radiation damage of microelectronics for spacecraft. Such missions require a monitoring device capable of measuring a wide range of particles and energies, whilst still adhering to constraints for space deployment

Manuscript received July 25, 2007; revised October 6, 2007. This work was supported by the National Space and Biomedical Research Institute through NCC 9-58. A. Wroe would like to acknowledge the support he received from the Australian American Fulbright Association and Australian Institute of Nuclear Science and Engineering (AINSE).

A. Wroe and A. Rosenfeld are with the Centre for Medical Radiation Physics, University of Wollongong, Wollongong, NSW 2087, Australia (e-mail: ajw16@ uow.edu.au; anatoly@uow.edu.au).

M. Reinhard is with the Australian Nuclear Science and Technology Organisation, Menai, NSW 2234, Australia (e-mail: mrz@ ansto.gov.au)

V. Pisacane, J. Ziegler, M. Nelson, and J. Dicello are with the United States Naval Academy, Annapolis, MD 21401 USA (e-mail: pisacane@usna.edu; ziegler@aya.yale.edu; nelson@usna.edu; dicello@usna.edu ).

F. Cucinotta is with the Radiation Research Department, NASA, Houston, TX 77058 USA (e-mail: francis.a.cucinotta@nasa.gov)

M. Zaider is with the Medical Physics Department, Memorial Sloan Kettering Cancer Care Center, New York, NY 10021 USA (e-mail: zaiderm@mskcc.org).

Digital Object Identifier 10.1109/TNS.2007.910037

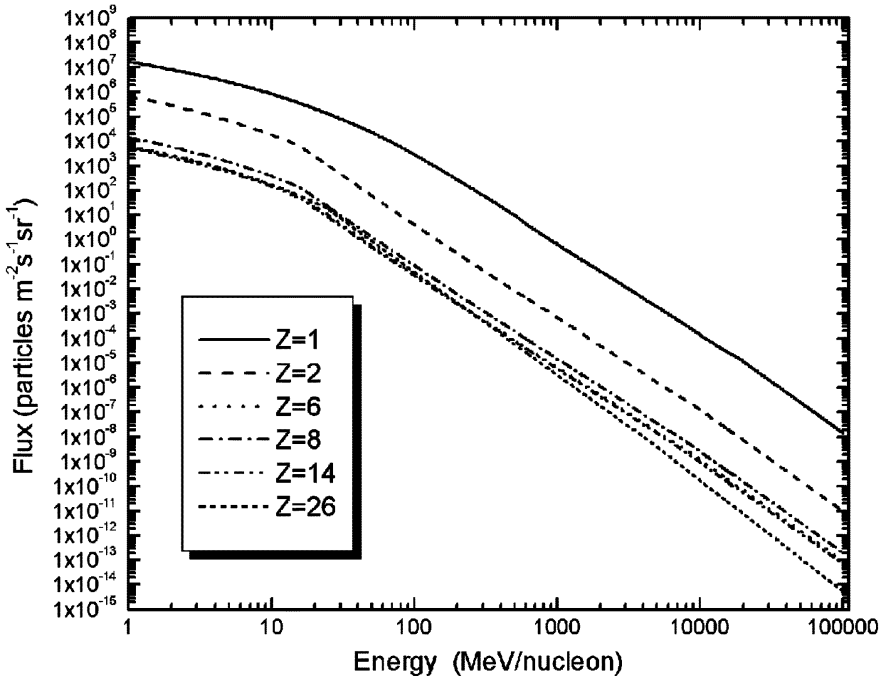

Fig. 1. Particle radiation spectra generated using CREME96 for a range of ions incident on the International Space Station [9].

such as low weight, low voltage of operation, low power consumption and low maintenance. Microdosimetry, which measures the radiation effects on a micron or cellular level producing a lineal energy spectrum is suitable for the measurement of such radiation fields as it can consider radiation interactions from a wide range of particles and energies [7]. When this spectrum is correlated with a well established quality spectra [8] it is possible to determine the dose equivalent.

The advantages of microdosimetry have led to the deployment of tissue equivalent proportional counters (TEPCs) [7] on near Earth space missions such as the International Space Station (ISS). TEPCs are the standard for microdosimetry as they provide an instrument large in volume (which is suitable for the low flux radiation environments of space), spherical in shape (hence the mean chord length is well defined) and tissue equivalent. However, TEPCs have the disadvantage of a being relatively sensitive instruments which can pose a problem for long term space deployment. A solid state microdosimeter is more rugged making it potentially more suitable for such deployments, and with the use of arrays of microscopically small SVs can also obtain data in a useful time frame from low flux radiation fields.

A Silicon-On-Insulator (SOI) microdosimeter (Fig. 2) that has been developed at the Center for Medical Radiation Physics (CMRP) at the University of Wollongong is a solid-state device which has been tested extensively in proton, neutron and heavy ion fields associated with cancer therapy [10]-[17]. In these 


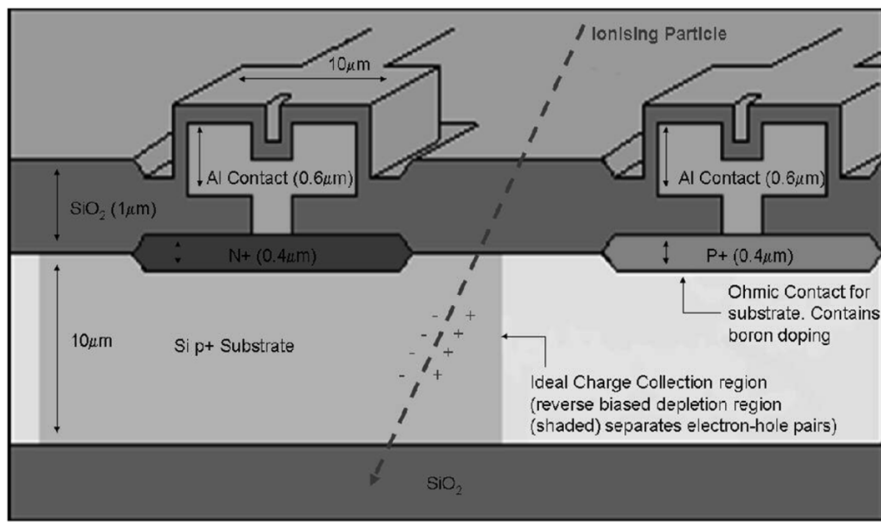

Fig. 2. Schematic cross-section of the SOI microdosimeter discussed in this article.

tests the device has proven itself to be a useful instrument in obtaining accurate lineal energy data for a range of particles and energies. The ability of this device to detect a range of particles, LET's and energies makes it suitable for a space deployment.

The device comprises of a 2D diode array of microscopically small sensitive volumes. On each chip there are three different sized arrays with sensitive volumes (SVs) of two different cross sectional areas; $100 \times 100 \mu \mathrm{m}^{2}$ and $30 \times 30 \mu \mathrm{m}^{2}$. Such a configuration allows for the solid-state device to be utilized in a wide range of radiation fields and fluxes avoiding signal pile-up. The other advantage of the present device is that it comes in three different silicon substrate thicknesses; 2, 5 and $10 \mu \mathrm{m}$, allowing a range of sensitive volume sizes to be deployed and compared when faced with a mixed radiation field. The advantages of such a system for space deployments has resulted in its inclusion as part of the dosimetry payload aboard the MidSTAR-1 satellite [18] and is a collaborative scientific effort headed by Prof. Vince Pisacane [19]. In this deployment three separate detectors were included within various configurations to measure the spectra of energy deposition events both external to and within the spacecraft structure.

The purpose of this work was to test the SOI microdosimeter under heavy ion radiation fields such as those experienced from GCR. In doing so this work provides the first experimental data using SOI microdosimetry in such heavy ion fields. The devices were tested at the National Space Radiation Laboratory (NSRL) at Brookhaven National Laboratory (BNL) using $0.6 \mathrm{GeV} / \mathrm{u}$ iron and $1.0 \mathrm{GeV} / \mathrm{u}$ titanium ions. Microdosimetry measurements were completed both at the surface and also at different depths within a Lucite phantom, simulating the change in microdosimetry spectra as it traverses a human body and behind shielding structures. Additionally, the measurement of the changing microdosimetry spectra of slowing incident ions provides information for a range of ion energies and for a greater portion of nuclear secondaries, better reflecting the spectra of particles observed in a space environment (Fig. 1). Such measurements were correlated with not only the depth dose curve of the incident radiation but also the dose mean lineal energy measurements made with a TEPC where available.

\section{METHOD: IRRADIATION FACILITY}

The NSRL facility has been specifically designed to study space radiation and its effects on biological and electronic systems. It utilizes the existing Brookhaven MP-6 tandem accelerator and Booster synchrotron to accelerate the ions to the required energies [20]. This facility allows for a wide range of ions and energies to be accelerated and experimented with providing a beam spill length of $300 \mathrm{~ms}$, with a total spill duration of $3.8 \mathrm{~s}$. The facility also allows for the production of uniform beam intensities of rectangular areas ranging in size from $1 \times 1$ to $20 \times 20 \mathrm{~cm}^{2}$. For this work the incident radiation field consists of $0.6 \mathrm{GeV} / \mathrm{u}$ iron-56 and $1 \mathrm{GeV} / \mathrm{u}$ titanium- 48 both in a fully stripped state. The field size used for this application was approximately 3-4 cm in diameter at FWHM with a frequency of $1-5 \mathrm{kHz}$ across field cross sectional area and was produced using the focusing magnets upstream of the experimental hall. As such, collimation of the field was not required, minimizing the level of secondary neutrons in the experimental hall. The advantage of this field size was that it minimized primary beam interaction with the associated electronics that are located in close proximity to the detector volume.

\section{Method: Phantom And Detectors}

A $5 \mu \mathrm{m}$ thick SOI microdosimeter chip was utilized in this study. The array chosen in this experiment comprises of 150 detector elements $(30 \times 5)$ with each sensitive volume of the cell defined by the effective volume of charge collection $100 \times$ $100 \mu \mathrm{m}^{2}$ which was measured experimentally using an alpha particle micro-beam. The geometrical size of cell planar $p-n$ junction was $120 \times 120 \mu \mathrm{m}^{2}$. The thickness of the microdosimeter SV is determined by the thickness of the bonded silicon used to manufacture of these SOI wafers and is known within a $0.5 \mu \mathrm{m}$ confidence. Verification of the thickness was completed previously using alpha particle spectroscopy under full depletion conditions [21].

The device was contained within a $0.9 \mathrm{~mm}$ thick probe holder of aluminum which acts as a Faraday cage. A $4 \mu \mathrm{m}$ aluminum window was located immediately in front of the microdosimeter to allow the transport of secondary particles into the SV (Fig. 3). This experimental assembly has been used in previous work with protons and neutrons and is described further in [16]. To enable reproducible placement of the microdosimeter probe in the centre of the radiation field and at various points within the Lucite phantom, it was housed within a specifically designed Lucite probe holder.

Located within the probe was the microdosimeter circuitry including an A-250 pre-amplifier, field-effect transistor (FET) and buffer amplifier $(\times 10)$. To minimize radiation interaction with the microdosimetry electronics, the beam size was limited to ensure uniform irradiation of the detector SV. Bias to the SOI microdosimeter (of $10 \mathrm{~V})$ and power to the A-250 $( \pm 6 \mathrm{~V})$ were supplied via a specifically designed battery supply which provided low noise. An energy calibration was applied to the results that were derived using a calibrated pulser, which in turn was calibrated using a $350 \mu \mathrm{m}$ thick planar Silicon detector of similar capacitance to the microdosimetry detector array and an Am-241 source. 


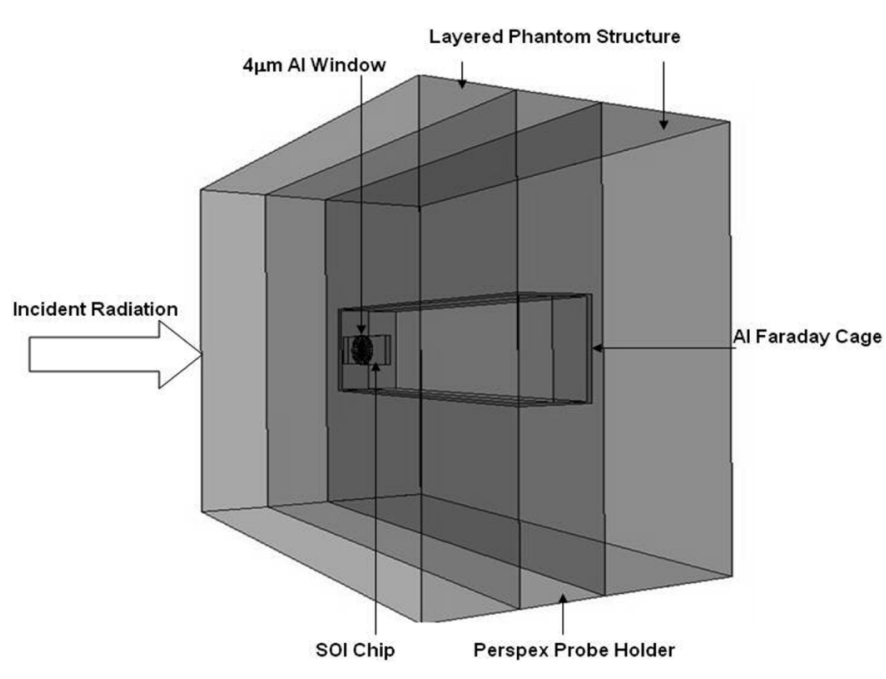

Fig. 3. Schematic diagram of the experimental setup. Note the layered Lucite structure enabling the sampling of the microdosimetric spectra at various depths and hence positions along the Bragg Peak.

Data acquisition was completed using a Tennelec Model 244 main amplifier and an Amptek Pocket Multi-Channel Analyzer (MCA). Regular noise level checks using a pulser were completed both pre, during and post experiment inside the experimental hall with the beam off. To minimize the effect background and electronic noise, the MCA was gated on a square logic pulse that surrounded the beam (i.e., $300 \mathrm{~ms}$ in length). All energy deposition events within the SOI microdosimeter were registered and as such no discrimination was made to consider only primary particles. Microdosimeters that are currently under development at the CMRP may allow for such discrimination in future experiments using particle identification techniques. All results obtained were normalized to a 100 second MCA live-time of acquisition.

Bragg curves in polyethylene were supplied by the staff at the NSRL facility and were obtained using high density polyethylene $\left(\rho=0.97 \mathrm{~g} / \mathrm{cm}^{3}\right)$ foils and $2 \times 32 \mathrm{~cm}$ diameter ion chambers (each consisting of 8 concentric rings at $2 \mathrm{n} \mathrm{cm}$ radii, where $n=1,2 \ldots, 8)$. One chamber was situated upstream of the phantom (for normalization) and the second situated downstream of a given thickness of phantom material. To obtain the Bragg Curve in Lucite the results were scaled according to phantom density as provided by NIST [22]. Such curves enabled accurate microdosimetry measurement positions to be determined and correlated with corresponding Bragg Peak position. They also allowed for the beam energy to be accurately determined using SRIM [23] and the range of the particles in polyethylene. For Fe the incident energy was determined to be $585.1 \mathrm{MeV} / \mathrm{u}$ (Fig. 4), while for Ti this was $977.8 \mathrm{MeV} / \mathrm{u}$ (Fig. 5). From these depth dose curves SOI measurement positions could be planned for, which would adequately sample the Bragg Peak and distal edge. The SOI microdosimeter measurement positions for Fe and Ti ions are displayed in Figs. 4 and 5, while the same labeling is used in the description of the results obtained at each position of measurement.

Generation of microdosimetry spectra and dose mean lineal energy was completed using the protocol outlined in ICRU 36

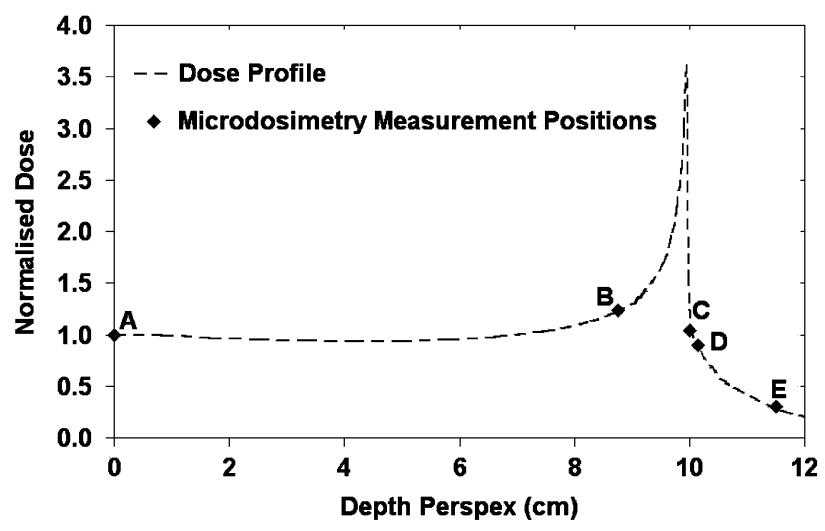

Fig. 4. Microdosimetry measurement positions along the $0.6 \mathrm{GeV} / \mathrm{u} \mathrm{Fe} \mathrm{Bragg}$ Peak in Lucite normalized to the entrance dose.

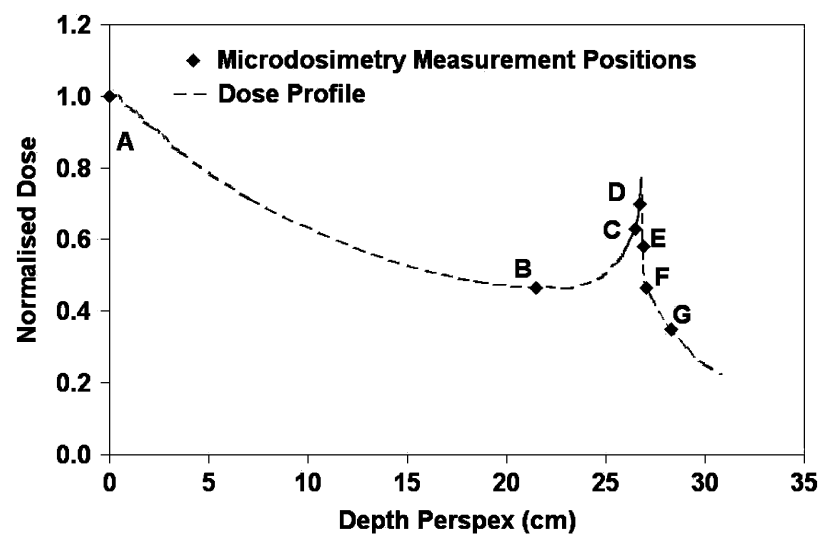

Fig. 5. Microdosimetry measurement positions along the $1.0 \mathrm{GeV} / \mathrm{u}$ Ti Bragg Peak in Lucite normalized to the entrance dose.

[8], [24]. In this study an array of $100 \times 100 \times 5 \mu \mathrm{m}^{3}$ geometrical SV elements was used. The mean chord length is determined by design of the SV and is defined in (1)

$$
<l>_{\mathrm{Si}}=\frac{4 V \alpha}{S} \quad<l>_{\mathrm{TE}}=\frac{4 V \alpha}{S \varsigma} .
$$

Equation (1): Definition of mean chord length, $\langle l\rangle$, in both $\mathrm{Si}$ and tissue from [24]. Note that $\mathrm{V}$ is the volume, $\mathrm{S}$ is the surface area, $\alpha$ is the CCE and $\zeta$ the TE conversion factor.

In this case the mean chord length was governed mainly by central part of $\mathrm{N}+$ region $10 \times 10 \mu \mathrm{m}$ and applied bias, which determines the charge collection efficiency (CCE or $\alpha$ ) in the lateral dimensions of the SV [25]. Increasing the geometrical size of SOI SV to $100 \times 100 \mu \mathrm{m}^{2}$ (from the more typically used $30 \times 30 \mu \mathrm{m}^{2}$ ) does not essentially change the mean chord length, as any increase in size is offset by a proportional reduction in average CCE. For a $100 \times 100 \times 5 \mu \mathrm{m}^{3}$ SOI volume the $\mathrm{CCE}$ is 0.55 , resulting in a mean chord length in $\mathrm{Si}$ of $5 \mu \mathrm{m}$ or the thickness of the SV. Conversion to the mean chord length in tissue requires the use of a scaling coefficient, $\zeta$. In previous work with the SOI microdosimeter such a scaling coefficient has been established as 0.63 [13], [21]. As such the mean chord length in tissue for the SV employed in this work was 7.936 $\mu \mathrm{m}$. This value was used for the generation of TE lineal energy spectra and dose mean lineal energy values. 


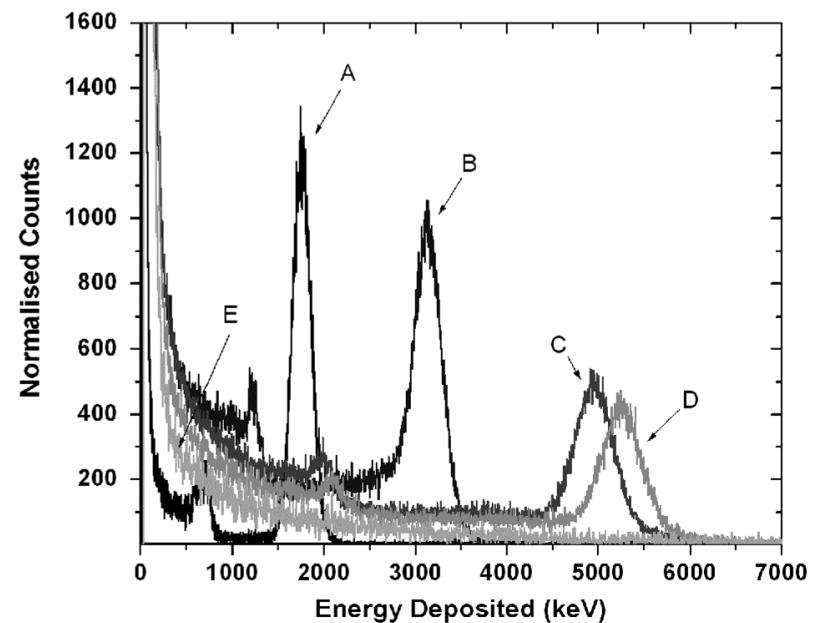

Fig. 6. $\mathrm{f}(\mathrm{E})$ spectra in $\mathrm{Si}$ for iron ions at the various measurement positions (A-E) in Lucite.

\section{RESUlTS AND DisCUSSION}

The SOI microdosimeter provided results on changing microdosimetric spectra of $0.6 \mathrm{GeV} / \mathrm{u}$ iron and $1.0 \mathrm{GeV} / \mathrm{u}$ titanium ions both in at the phantom surface and as they traverse a Lucite phantom. From the results in Fig. 6 the change of the iron peak energy with differing depth in Lucite is apparent. In the case of the surface measurement (position A), some interaction is observed below $800 \mathrm{keV}$ and this is most likely caused by lighter ions which are present in the radiation field, nuclear fragments and also through interaction of the Fe ions within the Si SV. The presence of energy depositions below the iron peak increases at depth due as a result of increased levels of secondary particle production through primary beam interaction with the phantom. A low energy peak is also observed, which is described and analyzed further in Section 5. It is also possible to observe the effect of straggling on the measurement, which is evidenced by widening of the Fe peak full width at half maximum (FWHM) with increasing depth in Lucite.

The inclusion of a measurement position past the distal edge of the Bragg peak illustrates the production of neutrons and other nuclear fragments both within the beam modifying/monitoring devices and also within the phantom itself that travel to greater depths within the phantom than the primary beam. The same energy spectra measured at $11.5 \mathrm{~cm}$ depth in Lucite (position E) is also present for all other measurement points (except at the surface of the phantom) suggesting that this was caused by secondaries produced from primary beam interactions within the phantom.

Converting the energy deposition spectra into a distribution of lineal energies provides further information on the radiation field (Fig. 7). These results provide a good indication on the change in lineal energy of a $0.6 \mathrm{GeV} / \mathrm{u} \mathrm{Fe}$ beam with increasing depth in Lucite as the main lineal energy peak moves to higher values. This also displays the functionality of the microdosimetry method in determining first lineal energy spectra and then dose mean lineal energy from simple energy deposition spectra $\mathrm{f}(\mathrm{E})$.

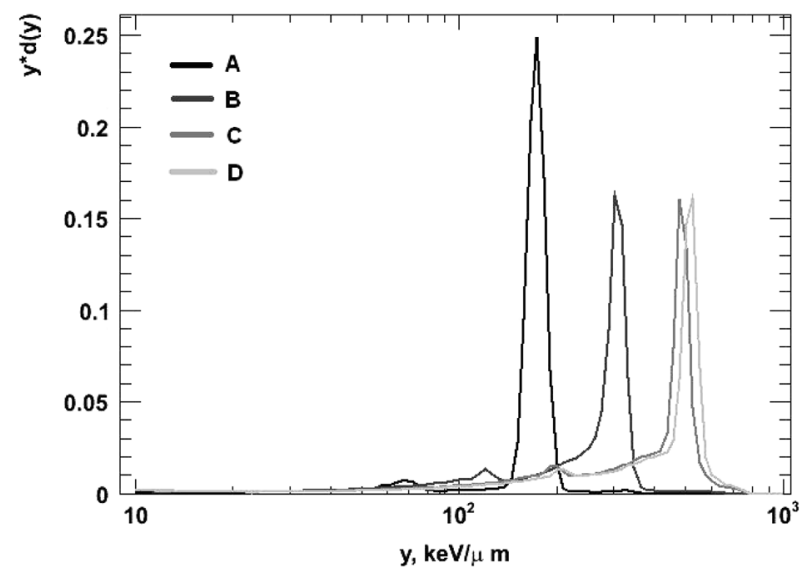

Fig. 7. TE Lineal energy spectra generated from experimental results for 0.6 $\mathrm{GeV} / \mathrm{u} \mathrm{Fe}$.

From the lineal energy spectra the dose mean lineal energy was calculated using the method outlined in (2)

$$
\begin{aligned}
\bar{y}_{d} & =\int_{0}^{\infty} y d(y) d y \\
& =\frac{1}{\bar{y}_{f}} \int_{0}^{\infty} y^{2} f(y) d y \\
\text { where } ; \bar{y}_{f}= & \frac{\int_{0}^{\infty} y f(y) d y}{\int_{0}^{\infty} f(y) d y} .
\end{aligned}
$$

Equation (2): Relationship describing the frequency mean lineal energy $\left(\bar{y}_{f}\right)$ and dose weighted mean lineal energy $\left(\bar{y}_{d}\right)$ where $\mathrm{y}$ is lineal energy [24].

The dose mean lineal energy can also be overlaid with the Bragg Curve to illustrate this change in relation to the Bragg Peak (Fig. 8). The measured lineal energy increases markedly near the Bragg Peak achieving maximum values of $479 \mathrm{keV} / \mu \mathrm{m}$ at the distal edge. Such increases could indicate increased single event upset (SEU) rate as the ionization density increases.

The Ti results (Fig. 9) are very similar to those previously described for $\mathrm{Fe}$. The titanium peak moves to higher energies with increasing depth in Lucite from a mean energy of $1.03 \mathrm{MeV}$ to approximately $5 \mathrm{MeV}$ on the distal edge of the Bragg peak. At the surface of the phantom some interaction is observed below $500 \mathrm{keV}$ and this is most likely caused by lighter ions which are present in the radiation field (produced through primary beam interactions with beam modifying and monitoring devices), and also through interaction of the Ti ions within the silicon SV. The presence of interactions below the Ti peak increases at depth due to the production of secondary particles through primary beam interaction with the phantom. As for $\mathrm{Fe}$, a secondary peak is observed for the surface measurement, however this is largely masked at depth due to the increase of signal below the main energy deposition peak. This is discussed further in Section 5. Secondary particles such a neutrons also contribute to signal past the Bragg peak (measurement position $\mathrm{G}$ ). 


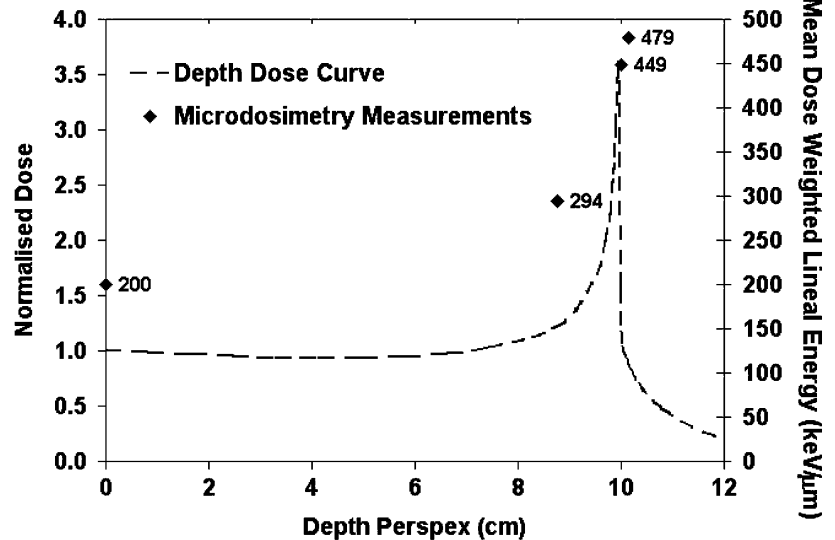

Fig. 8. TE Dose weighted lineal energy values plotted against the depth dose curve of $0.6 \mathrm{GeV} / \mathrm{u} \mathrm{Fe}$.

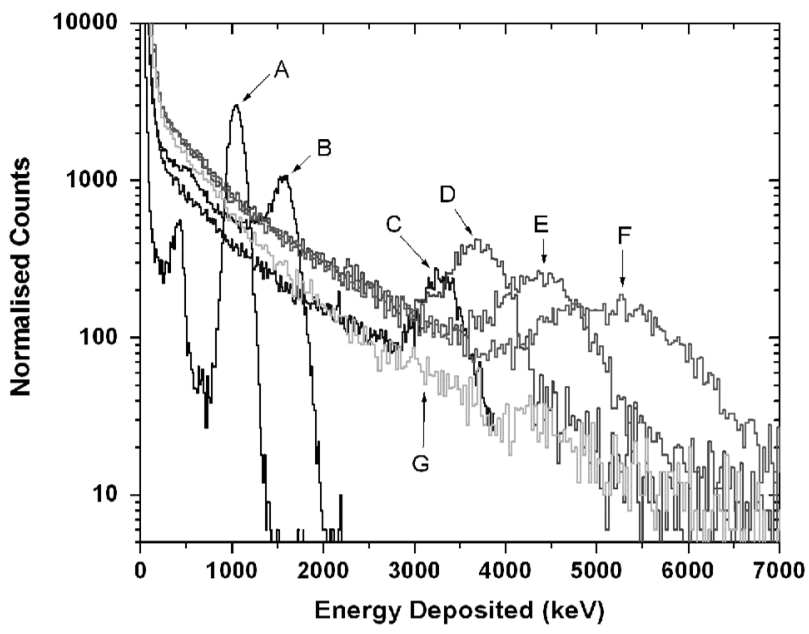

Fig. 9. $f(E)$ spectra in $\mathrm{Si}$ for titanium ions for the various measurement positions (A-G) in Lucite.

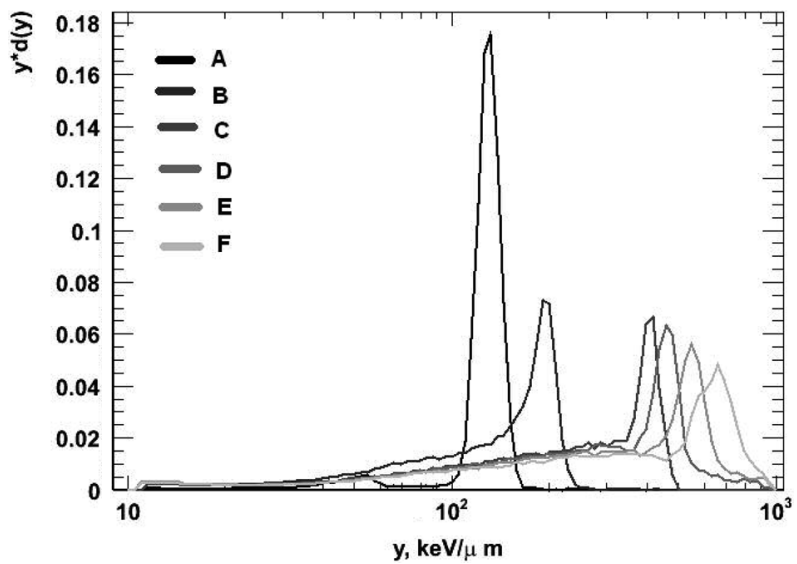

Fig. 10. TE lineal energy spectra as determined from the experimental results for $1.0 \mathrm{GeV} / \mathrm{u} \mathrm{Ti}$.

Converting the energy deposition spectra into a distribution of lineal energies provides a good indication of the change in lineal energy of a $1.0 \mathrm{GeV} / \mathrm{u}$ Ti beam with depth in Lucite (Fig. 10). Increasing depth in Lucite causes the main peak to move to higher lineal energy values, while the increase in lower energy deposition events has only a minimal effect on the spectra which is

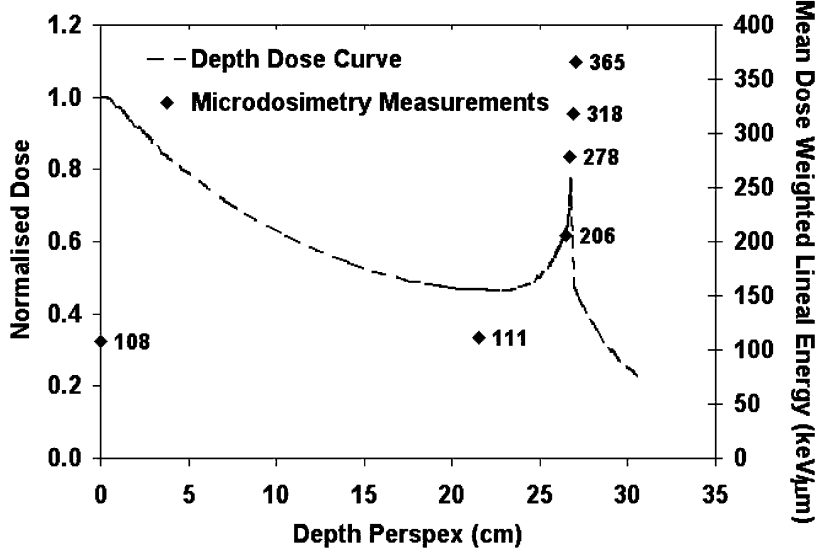

Fig. 11. TE dose weighted lineal energy values plotted against the depth dose curve of $1.0 \mathrm{GeV} / \mathrm{u} \mathrm{Ti}$.

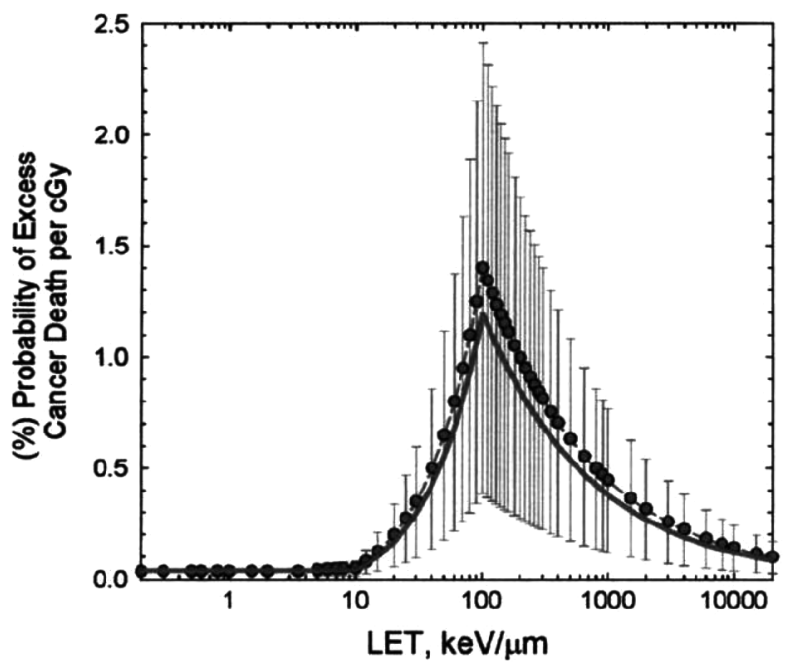

Fig. 12. Calculation of the point estimate (symbols with dash line) and median values (solid lines) for the probability of excess fatal cancer per cGy versus LET and their subjective standard errors [3].

indicative of the weighting provided by microdosimetry to high lineal energy events which can be potentially more damaging to electronic structures.

When comparing the dose mean lineal energy of each measurement point with the depth dose profile of Ti (Fig. 11) it is clear that the measured lineal energy increases markedly from $108-365 \mathrm{keV} / \mu \mathrm{m}$ near the Bragg peak achieving maximum values at the distal edge. Such results reflect the same trend as that of Fe ions with the dose mean lineal energy increasing as the ion slows and the delta electron track structure density increases. However, the absolute value of the dose mean lineal energy is different between the two ions as a result of an inherently different secondary electron track structure between the two different ion species.

The advantage of the microdosimetry approach is that it can be used to directly determine dose equivalent using established quality factor distributions [26]. Mean lineal energy can also be linked to biological predictions to provide a possible estimation cell death and mutation rates. In Fig. 12 the calculation of the probability of excess cancer with varying LET is presented. A 
trend is discernable as a function of particle LET displaying a peak at $100-200 \mathrm{keV} / \mu \mathrm{m}$ which corresponds to Fe and Ti particles in the energy range measured here. However, risk projections such as these involve a product of many biological and physical factors and as data on many of these factors is limited, the errors associated with such risk estimates can be large. Examples of such uncertainties include the type, energy and fluence of the incident radiation field as well as a limitation of our understanding of how such particles affect human tissues. Despite this, the use of such measured values may allow for the optimizing of shielding structures to avoid these regions of lineal energy and possibly result in a reduction in the incidence of fatal cancer induction.

\section{Low ENergy PeaK ANALysis}

The low energy peak detected in heavy ion SOI microdosimetry studies is clearly apparent in the energy deposition spectra collected using Fe-56 (Fig. 7) and Ti-48 (Fig. 10) ions. Charge collection or "fringing" outside the SV volume array was investigated using Ion Beam Induced Charge Collection Imaging (IBICC) and may contribute, in part, to this formation. However, the fringing effect was observed at approximately $5 \%$ charge collection, or approximately $10 \%$ of the full energy peak for incident He particles in IBICC. It would be reasonable to expect the same charge division effect in the same array of the SOI microdosimeter for Fe and Ti ions. Especially as the incident alpha particle used in IBICC studies were of a similar stopping power to ions studied at NSRL. However, in the case of $\mathrm{Fe}$ and Ti measurements, the secondary peak occurs at approximately $40 \%$ and $45 \%$ of the full energy deposition peak respectively. It was deemed that while the fringing effect may contribute to the low energy signal detected by the microdosimeter, it was possible that this effect may be caused by Si recoils generated through elastic nuclear interactions within the detector volume. Such effects have not been observed previously in studies with this device as the ions studied were sufficiently light, or of sufficiently low energy to either prevent production of such recoils or the recoils produced were below the low noise threshold of the device. The maximum energy delivered to a stationary particle, in this case a Si nucleus, from an incident ion is described in (3)

$$
E_{s i}=\frac{4 \times M_{s i} \times M_{x}}{\left(M_{s i} \times M_{x}\right)^{2}} \times E_{x} .
$$

Equation (3): Relationship for maximum energy transfer between an incident ion of species $X$, and a Si nucleus via elastic nuclear interaction. $\mathrm{E}_{S} i$ and $\mathrm{M}_{S} i$ is the energy and mass of the recoil Si nucleus and $\mathrm{E}_{X}$ and $\mathrm{M}_{X}$ is the energy and mass of the incident ion of species $X$ [27].

In this case the maximum energy of the Si recoil will be 0.88 the energy of an incident Fe ion and 0.93 the energy of an incident Ti ion. For the measurements at the surface of the phantom, where the incident particle has not undergone significant attenuation, the maximum energy of the Si recoil will be 29.57 and $44.64 \mathrm{GeV}$ for incident $0.6 \mathrm{GeV} / \mathrm{u} \mathrm{Fe}$ and $1.0 \mathrm{GeV} / \mathrm{u}$ Ti respectively. Using SRIM the relative stopping powers of the ions in Si were determined and are contained in Table I.
TABLE I

COLLECTION OF INFORMATION ON THE STOPPING POWER IN SILICON PROVIDED BY SRIM [23] FOR INCIDENT FE AND TI PARTICLES USED IN HEAVY ION MICRODOSIMETRY WORK AND ASSOCIATED SI RECOILS

\begin{tabular}{ccc}
\hline \hline ION SPECIES & ENERGY $(\mathrm{GEV})$ & STOPPING POWER $(\mathrm{KEV} / \mu \mathrm{M})$ \\
\hline \hline $\mathrm{Fe}$ & 33.60 & 318.76 \\
$\mathrm{Ti}$ & 48.00 & 198.23 \\
$\mathrm{Si}$ (generated by Fe) & 29.57 & 80.67 \\
$\mathrm{Si}$ (generated by $\mathrm{Ti})$ & 44.64 & 76.31 \\
\hline \hline
\end{tabular}

TABLE II

TABULATED DATA ON THE ENERGY AND LINEAL ENERGY OF THE MAIN AND SECONDARY PEAKS CONTAINED Within the EXPERIMENTALLY MEASURED SOI ENERGY SPECTRUM FOR INCIDENT FE AND TI IONS

\begin{tabular}{cccc}
\hline INCIDENT ION & PEAK & ENERGY (KEV) & $\begin{array}{c}\text { LINEAL ENERGY } \\
(\mathrm{KEV} / \mu \mathrm{M})\end{array}$ \\
\hline \hline Iron & Primary & 1750 & 350 \\
Iron & Secondary & 710 & 142 \\
Titanium & Primary & 1050 & 210 \\
Titanium & Secondary & 450 & 90 \\
\hline \hline
\end{tabular}

From the experimentally measured data it was possible to determine the lineal energy in Silicon (using a chord length of 5 $\mu \mathrm{m})$ for both the main ion peak and the secondary peak in question. This information is contained within Table II. The stopping power information obtained from SRIM is in good agreement with the experimentally derived data for the primary ion energy deposition peak, further supporting the use of SOI microdosimeters in heavy ion measurements.

The lineal energy of the secondary peak closely reflects that of the stopping power for Si recoils of maximum theoretical energy imparted by incident $\mathrm{Fe}$ and $\mathrm{Ti}$ ions used in this experiment and derived using (3) and SRIM. There is some difference between the lineal energy value of the secondary peak in the experimental spectrum and the stopping power of the $\mathrm{Si}$ recoil of maximum energy. For Ti this is relatively small with a discrepancy of $18 \%$, while for Fe generated Si recoils this error is approximately $75 \%$. These discrepancies can be attributed to the Si recoil energy having a dependence on the angle of recoil, and also on the point of interaction within the SV.

It is also important to remember that the energy imparted to a recoil nucleus is dependant on the relative charge states of the incident and target ions. In this case the incident ion whilst fully ionised in the vacuum tube does traverse a number of beam windows, monitoring chambers and air before entering the SV of the microdosimeter. The traversing of these materials may cause the charge state of the ion to change hence reducing the maximum energy which it can impart on the Si nucleus. The charge state of the ion as it enters the SOI microdosimeter can not be determined from current data and as such is a source of inaccuracy in this estimation.

The fact that the position of the peak is reflected in the stopping power of Si recoils with theoretical maximum energy supports the theory that this is the cause of the secondary peak in the heavy ion energy spectra. Energy depositions below this secondary peak can be attributed to:

- Si recoils being imparted energy less than the maximum transferable via an elastic collision with an incident particle. 
- Other particles which make up the incident beam interacting within the SV.

- The fringing effect of charge collection, which is an inherent property of the current SOI technology.

Further work in this regard is currently underway utilizing Monte Carlo simulation programs.

\section{VERIFICATION OF RESULTS}

At the surface of the phantom the SOI microdosimeter measured a dose mean lineal energy of $200 \mathrm{keV} / \mu \mathrm{m}$ for 0.585 $\mathrm{GeV} / \mathrm{u} \mathrm{Fe}$ using a TE correction factor of 0.63 . This compares well with a TEPC measured values of $180 \mathrm{keV} / \mu \mathrm{m}$ for 0.535 $\mathrm{GeV} / \mathrm{u} \mathrm{Fe}$ [28] and $173 \mathrm{keV} / \mu \mathrm{m}$ for $0.540 \mathrm{GeV} / \mathrm{u} \mathrm{Fe} \mathrm{[29].}$ Considering that these were the first measurements with SOI microdosimetry technology in heavy ion fields of this energy, to be within $10-15 \%$ of existing data supports the further use and testing of such a system for deep space deployment. The difference in this case can be attributed to a number of factors concerning both the SOI microdosimeter measurement apparatus and the incident beam. Firstly the TEPC measurements were completed at the Bevalac Accelerator of the Lawrence Berkeley Laboratory CA [28] and the Alternating Gradient Synchrotron (AGS) at Brookhaven National Laboratory [29], while SOI microdosimetry measurements were completed at the NSRL facility located at Brookhaven National Laboratory. Different accelerator facilities have in place different beam monitoring/modifying devices which can contribute to a different spectrum of secondary particles. The spectra of secondary particles in such heavy ion experiments can be significant (as evidenced in [30]) and can influence microdosimetry measurements.

Solid-state microdosimetry measurements in a heavy ion field required certain assumptions to be made as these were initial measurements with SOI technology. These assumptions need to be considered when assessing differences to existing heavy ion data obtained using different measurement techniques (in this case a TEPC). The first assumption made was the average chord length. In this instance a relatively large planar microdosimeter was utilized that had a cross sectional area of $100 \times 100 \mu \mathrm{m}^{2}$ with a SV thickness of $5 \mu \mathrm{m}$. While the variance of chord lengths with the planar structure was considered, variation in this response may be encountered at increasing depth in the phantom and with differing levels of primary fragmentation and variation in the secondary particle spectra. To determine the effect of this assumption, GEANT4 Monte Carlo simulations should be completed to assess the mean chord length in such a field. Further, in the case of space deployment a more cubic structure would be more suitable, as in an omnidirectional field it will produce a more uniform response and be less dependant SV geometry.

The second assumption and possibly the main source of error in these measurements arise from the TE conversion factor. It has been shown in previous work with protons and alpha particles that the value of 0.63 gives good agreement to TEPC devices, however this has yet to be tested using heavy ions such as Fe-56. With agreement to established data being within $12 \%$ (which is in-line with previous estimations that the thickness of the SOI has a variation of $\pm 10 \%$ ) it can be assumed that this has been a fair assumption, and can be further proved through the use of Monte Carlo simulations.

\section{CONCLUSION}

This work provided information on the performance of the SOI microdosimeter in heavy ion radiation fields. The results of this device compare well with established TEPC data and illustrate how such a device is useful in determining lineal energy data within phantoms and behind shielding structures. The design features and performance of this detector in this work supports its further testing and deployment in a space environment.

\section{ACKNOWLEDGMENT}

The authors would like to thank Adam Rusek and Mike Sivertz for their invaluable support during experiments at NSRL. Further, our collaborators at the Australian Nuclear Science and Technology Organisation and the United States Naval Academy were supportive in the development and testing of the experimental system.

\section{REFERENCES}

[1] F. A. Cucinotta and J. F. Dicello, "On the development of biophysical models for space radiation risk assessment," Adv. Space Res., vol. 25, pp. 2131-2140, 2000.

[2] F. A. Cucinotta, W. Schimmerling, J. W. Wilson, L. E. Peterson, G. D. Badhwar, P. B. Saganti, and J. F. Dicello, "Space radiation cancer risks and uncertainties for Mars missions," Radiati. Res., vol. 156, pp. 682-688, 2001.

[3] F. A. Cucinotta, W. Schimmerling, J. W. Wilson, L. E. Peterson, P. B. Saganti, and J. F. Dicello, "Uncertainties in estimates of the risks of late effects from space radiation," Adv. Space Res., vol. 34, pp. 1383-1389, 2004.

[4] F. A. Cucinotta, J. W. Wilson, and J. F. Dicello, "Physics and biophysics problems in cosmic ray exposures and risk assessment," $R a$ diat. Effects Defects Solids, vol. 141, pp. 457-457, 1997.

[5] J. F. Dicello, "The impact of the new biology on radiation risks in space," Health Phys., vol. 85, pp. 94-102, 2003.

[6] J. A. Simpson, Introduction to the Galactic Cosmic Radiation. Composition and Origin of Cosmic Rays. Amsterdam, The Netherlands: Reidel, 1983.

[7] G. D. Badhwar, "Shuttle radiation dose measurements in the international space station orbits," Radiat. Res., vol. 157, pp. 69-75, 2002.

[8] H. H. Rossi and M. Zaider, Microdosimetry and Its Applications. New York: Springer-Verlag, 1996.

[9] CREME96 [Online]. Available: https://creme96.nrl.navy.mil

[10] A. J. Wroe, I. M. Cornelius, A. B. Rosenfeld, V. L. Pisacane, J. F. Ziegler, E. Nelson, F. Cucinotta, M. Zaider, and J. F. Dicello, "Microdosimetry simulations of solar protons within a spacecraft," IEEE Trans. Nucl. Sci., vol. 52, no. 6, pp. 2591-2596, Dec. 2005.

[11] P. D. Bradley and A. B. Rosenfeld, "Tissue equivalence correction for silicon microdosimetry detectors in boron neutron capture therapy," Med. Phys., vol. 25, pp. 2220-2225, 1998.

[12] P. D. Bradley, A. B. Rosenfeld, B. Allen, J. Coderre, and J. Capala, "Performance of silicon microdosimetry detectors in boron neutron capture therapy," Radiat. Res., vol. 151, pp. 235-243, 1999.

[13] P. D. Bradley, A. B. Rosenfeld, and M. Zaider, "Solid state microdosimetry," Nucl. Instrum. Methods Phys. Res. B, vol. B184, pp. 135-157, 2001.

[14] A. B. Rosenfeld, P. D. Bradley, I. Cornelius, B. J. Allen, M. Zaider, R. L. Maughan, J. C. Yanch, J. Coderre, J. B. Flanz, and T. Kobayashi, "Solid state microdosimetry in hadron therapy," Radiat. Protect. Dosimetry, vol. 101, pp. 431-434, 2002.

[15] A. B. Rosenfeld, P. D. Bradley, I. Cornelius, G. I. Kaplan, B. J. Allen, J. B. Flanz, M. Goitein, A. Van Meerbeeck, J. Schubert, J. Bailey, Y. Takada, A. Maruhashi, and Y. Hayakawa, "A new silicon detector for microdosimetry applications in proton therapy," IEEE Trans. Nucl. Sci., vol. 47, no. 4, pp. 1386-1394, Aug. 2000. 
[16] A. Wroe, A. Rosenfeld, I. Cornelius, D. Prokopovich, M. Reinhard, R. Schulte, and V. Bashkirov, "Silicon microdosimetry in heterogeneous materials: Simulation and experiment," IEEE Trans. Nucl. Sci., vol. 53, no. 6, pp. 3738-3744, Dec. 2006.

[17] A. Rosenfeld, A. Wroe, M. Carolan, and I. Cornelius, "Method of monte carlo simulation verification in hadron therapy with non-tissue equivalent detectors," Radiat. Protect. Dosimetry, vol. 119, pp. 487-490, 2006.

[18] V. L. Pisacane, Q. E. Dolecek, F. Maas, M. E. Nelson, P. J. Taddei, Z. Zhao, J. F. Ziegler, P. C. Acox, M. Bender, J. D. Brown, T. Garritsen, C. Gaughan, A. Hough, B. Kolb, J. Langlois, J. Ross, M. Sheggeby, D. Thomas, J. F. Dicello, F. A. Cucinotta, M. Zaider, A. B. Rosenfeld, and A. Wroe, "MIcroDosimeter iNstrument (MIDN) on MidSTAR-I," SAE Trans. J. Aerospace, vol. 2006-01-2146, 2006.

[19] MIDSTAR [Online]. Available: http://web.ew.usna.edu/midstar 2007

[20] NASA Space Radiation Facility [Online]. Available: http://server.c-ad. bnl.gov/esfd/nsrl/index.html

[21] P. Bradley, "The Development of a Novel Silicon Microdosimeter for High Let Radiation Therapy," Ph.D. dissertation, Engineering Physics Dept., Univ. Wollongong, Wollongong, Australia, 2000.

[22] National Institute of Standards and Technology Composition Database Program [Online]. Available: http://physics.nist.gov/cgi-bin/Star/ compos.pl?ap NIST
[23] J. F. Ziegler and J. P. Biersack, SRIM [Online]. Available: http://www. srim.org/\#SRIM 2003

[24] ICRU, vol. 36, 1983, Microdosimetry.

[25] P. D. Bradley, A. B. Rosenfeld, K. K. Lee, D. N. Jamieson, G. Heiser and S. Satoh, "Charge collection and radiation hardness of a SOI microdosimeter for medical and space applications," IEEE Trans. Nucl. Sci., vol. 45, no. 6, pp. 2700-2710, Dec. 1998

[26] International Commission on Radiation Units and MeasurementsThe Quality Factor in Radiation Protection Rep. ICRU-40, 1986.

[27] J. E. Turner, Atom, Radiation and Radiation Protection. New York: Pergamon, 1986.

[28] J. F. Dicello, M. Wasiolek, and M. Zaider, "Measured microdosimetric spectra of energetic Ion-Beams of $\mathrm{Fe}, \mathrm{Ar}, \mathrm{Ne}$, and $\mathrm{C}-\mathrm{Lim}-$ itations of let distributions and quality factors in space research and radiation effects," IEEE Trans. Nucl. Sci., vol. 38, no. 6, pp. 1203-1209, Dec. 1991.

[29] B. B. Gersey, T. B. Borak, S. B. Guetersloh, C. Zeitlin, J. Miller, L. Heilbronn, T. Murakami, and Y. Iwata, "The response of a spherical tissue-equivalent proportional counter to iron particles from 200-1000 MeV/nucleon," Radiat. Res., vol. 157, pp. 350-360, 2002.

[30] C. Zeitlin, S. B. Guetersloh, L. H. Heilbronn, and J. Miller, "Measurements of materials shielding properties with $1 \mathrm{GeV} / \mathrm{nuc} \mathrm{Fe}-56$," Nucl. Instrum. Methods Phys. Res. B, vol. B252, pp. 308-318, 2006. 